(Aus der Biologischen Station Lunz.)

\title{
Uferflucht des Planktons und ihr Einfluß auf die Ernährung von Salmonidenbrut.
}

\author{
Von \\ Dr. F. Ruttner (Lunz).
}

Bekanntlich zeigen die Plankton-Crustaceen insbesondere vieler Alpenseen die merkwürdige Eigentümlichkeit, die Uferregion zu meiden. Die Tiefe, über welcher sich in zahlreichen Gewässern schon eine Abnahme des Crustaceenplanktons bemerkbar inacht, ist eine überraschend große; so können wir im Lunzer Untersee die Isobathe von etwa $15 \mathrm{~m}$ als die äußere Grenze der limnetischen Region betrachten, von da ab gegen das Ufer hin verringert sich die Anzahl der Crustaceen zusehends, und manche Arten verschwinden bald vollständig. Aber nicht nur die Menge der plank. tonischen Kruster, die man in dieser Region erbeutet, ist eine geringe, es fehlen auch mehr oder weniger die litoralen Formen, wofern man sich beim Fischen oder Pumpen vom Bodengrund und der Vegetation etwas fern hält. Die Uferformen, soweit sie das Litoral des Lunzer Sees bevölkern, sind eben meist an das Substrat gebunden und schwärmen nicht weit in das sie umgebende Wasser aus.

Wir haben also zwischen der von Planktonkrustern bevölkerten limnetischen Region einerseits und dem Schlammgrund sowie den Pflanzenformationen der Uferbänke und der Seehalde mit ihrer reichen tierischen Bewohnerschaft andererseits einen Gürtel eingeschaltet, den man im fischereilichen Sinne als sehr nahrungsarm bezeichnen muß.

Es lag nun der, Gedanke nahe, diese Verhältnisse durch Aussetzen von Fischbrut in die soeben unterschiedenen Regionen praktisch zu prüfen. Derartige Experimente wurden in Lunz in den Jahren 1912 und 1913 in 'Angriff genommen, und, obwohl sie mehr den Cha- 
rakter von Vorversuchen trugen, und noch nicht als abgeschlossen zu betrachten sind, zeitigten sie doch einige Ergebnisse, die mir klar genug zu sein scheinen, um hier in Kürze besprochen werden zu können.

Es wurden für diese Versuche bisher ausschließlich Jungfische von Forellen und Saiblingen verwendet, die nach künstlicher Befruchtung in Blechbrutkästen erbrütet worden waren und nach Aufzehrung des Dottersackes zur Aussetzung kamen. Um für Parallelversuche ein möglichst gleichartiges Material zu erhalten, wurden hierfür soweit als möglich Abkömmlinge eines und desselben Elternpaares verwendet. Sie fanden in Holzkästen Aufnahme, welche $150 \mathrm{~cm}$ lang, $86 \mathrm{~cm}$ breit und $62 \mathrm{~cm}$ tief waren, und deren Boden sowie auch die Seitenwände, soweit sie ins Wasser tauchten, aus schlitzförmig gelochtem Zinkblech bestanden.

Diese „Kinderstuben“, welche jenen, die zur Aufzucht von Forellenbrut in Bächen seit langer Zeit verwendet werden, sehr ähnlich sind, wurden auf Flößen so befestigt, daß sie $37 \mathrm{~cm}$ ins Wasser tauchten, und in einem Falle mit je 150, im anderen mit je 100 Jungfischen beschickt, eine im Verhältnis zu dem geringen Fassungsraum (nicht ganz $1 / 2 \mathrm{cbm}$ ) recht starke Besetzung.

Im Frühling 1912 wurden nun vier solche Kästen am Ostufer des Untersees verankert, und zwar zwei am Rande eines ganz schütteren Schoenoplectus-Bestandes bei etwa 11/2 $\mathrm{m}$ Tiefe, $z$ wei weiter draußen bei etwa $20 \mathrm{~m}$ Tiefe. An beiden Punkten wurde je einer dieser Behälter mit 150 Forellen, der andere mit ebensovielen Seesaiblingen besetzt. Sowohl beim Aussetzen als auch an zwei späteren Zeitpunkten wurden je zehn der Fischchen herausgefangen und noch lebend gewogen. Die Ergebnisse dieser Wägungen sind in folgender Tabelle zusammengestellt:

\begin{tabular}{c||c|c|c|c}
\hline \multicolumn{1}{|c||}{} & \multicolumn{2}{c|}{ See } & \multicolumn{2}{c}{ Uf er } \\
\cline { 2 - 5 } & Forelle & Saibling & Forelle & Saibling \\
\hline \hline 20.V. beim Aussetzen & $1,1 \mathrm{~g}$ & $0,82 \mathrm{~g}$ & $1,1 \mathrm{~g}$ & $0,82 \mathrm{~g}$ \\
21. VI. & $2,13 \mathrm{~g}$ & $2,15 \mathrm{~g}$ & $1,13 \mathrm{~g}$ & $0,9 \mathrm{~g}$ \\
28. VIII. & $8,4 \mathrm{~g}$ & $6,7^{1} \mathrm{~g}$ & ausgestorben
\end{tabular}

1) Die Saiblinge wurden leider Ende August durch eine Ichthyophthirius-Epidemie fast vollständig vernichtet. Am 28. VIII. fanden sich nur mehr 2 Exemplare vor, der Wert von $6,7 \mathrm{~g}$ ist also nach der Wägung der 2 Exemplare für 10 Stück berechnet. 
Die Fische am Ufer nahmen sehr rasch an Zahl ab, und ihr Gewicht hatte sich nach einem Monat kaum vergrößert. Jene in der pelagischen Region des Sees dagegen waren in der gleichen Zeit doppelt so schwer geworden. Später starben die Versuchskästen am Ufer - bald aus, so daß eine weitere Gewichtsbestimmung nicht mehr möglich war. Die anderen zeigten auch weiterhin ein erfreuliches Wachstum, insbesondere die Forellen, während die Saiblinge, wie in der Anmerkung erwähnt, einer Krankheit zum Opfer fielen.

Hand in Hand mit diesen Gewichtsbestimmungen gingen Untersuchungen des Darminhaltes. Je drei der gewogenen Fische wurden geöffnet und der Darminhalt mikroskopisch geprüft. Die dabei gefundenen Unterschiede waren sehr lauffallend und stimmten mit den Ergebnissen der Wägungen gut überein: In den Fischen vom See draußen große Mengen von Planktonkrebsen, in jenen vom Ufer sehr vereinzelte litorale Crustaceen, in einigen Fällen ein paar Bosminen, Chitinteile von Insektenlarven, Eier von Insekten.

War es nach diesen Versuchen schon sehr wahrscheinlich, daß die in der Uferregion ausgesetzten Fische in verhältnismäßig kurzer Zeit an Nahrungsmangel zugrunde gehen, so könnte man doch vielleicht einwenden, daß diese Erscheinungen auf andere Ursachen, etwa auf die mitunter höhere Temperatur über der Uferbank zurückgeführt werden könnten.

Es wurde daher im nächsten Sommer (1913) eine neue Versuchsreihe eingeleitet, und zwar diesmal nur mit Forellenbrut. Von vier mit je 100 Fischen beschickten Kästen wurden in der oben beschriebenen Weise "zwei unter gleichen Verhältnissen bei $11 / 2 \mathrm{~m}$, einer bei 6-8 $\mathrm{m}$, der letzte schließlich bei etwa $18 \mathrm{~m}$ Tiefe verankert. Von den beiden Kästen lam Ufer wurde der eine täglich mit im See gefischtem Plankton versorgt, die Fische im anderen Kasten blieben jedoch ungefüttert. Folgende Tabelle zeigt die Resultate der Wägungen :

Lebendgewicht von je 10 Stück. 6. VI. beim Aussetzen: 0,88 g.

\begin{tabular}{c||c|c|c|c}
\hline \hline \multirow{2}{*}{} & \multicolumn{2}{|c|}{ Ufer } & \multirow{2}{*}{ über $6-8 \mathrm{~m}$} & über $18 \mathrm{~m}$ \\
\cline { 2 - 5 } & ungefüttert & gefüttert & & \\
\hline \hline 10. VII. & $1,2 \mathrm{~g}$ & $1,45 \mathrm{~g}$ & $1,43 \mathrm{~g}$ & $2,5 \mathrm{~g}$ \\
ungefähr noch vorhanden & 15 & 50 & 80 & 80 \\
25. VIII. & ausgestorben & $6,6 \mathrm{~g}$ & $3,7 \mathrm{~g}$ & $\mathbf{5 , 7 \mathrm { g }}$ \\
ungefähr noch vorhanden & - & 30 & 60 & 60
\end{tabular}


Aus diesen Versuchen geht hervor, daß durch eine Fütterung mit Plankton auch am Ufer ein erhebliches Wachstum erzielt und ein Absterben hintangehalten werden kann. Bei der ersten Beobachtung am 10. Juli trat dies noch nicht so deutlich hervor, und das Gewicht der gefütterten Fische am Ufer blieb hinter dem jener im Limneticum ausgesetzten weit zurück. Später jedoch zeigten die ersteren ein sehr lebhaftes Wachstum und überflügelten darin alle anderen. Dies dürfte allerdings zum Teil auch auf den Umstand zurückzuführen sein, daß anfangs aus einem mir unbekannten Grunde eine Abnahme der Anzahl auch unter den mit Plankton gefütterten Fischen am Ufer Platz griff, so daß für die Fortsetzung des Versuches nach dem 10. Juli nur mehr etwa die Hälfte übrigblieb, während die Tiere draußen im See noch fast vollzählig vorhanden waren. Es muß daher bei der Beurteilung des späteren, so außerordentlich lebhaften Wachstums auch die relativ schwächere Besetzung des betreffenden Behälters berücksichtigt werden.

Sonst zeigte sich auch in diesem Versuch sehr schön die Übereinstimmung der Gewichtszunahme der in verschiedenen Regionen ausgesetzten Fische mit der horizontalen Planktonverteilung, indem die über $6-8 \mathrm{~m}$ Tiefe befindlichen Tiere in ihren Zuwachsverhältnissen eine Mittelstellung zwischen den beiden Extremen am Ufer und in der ausgesprochenen Planktonregion einnahmen.

Leider wurde auch dieser Versuch im Spätsommer durch eine Epidemie vernichtet. Es scheint, idaß in Behältern, die an der Oberfläche schwimmen, starker Wellengang schädigend wirkt; auch die Oberflächentemperatur mag die Tiere ungünstig beeinflussen. Es spricht für diese Auffassung das gute Ergebnis, das ich durch Versenken von Fischen in größere Tiefen erzielte.

Anfang Juli 1912 wurden von den im Limneticum ausgesetzten Fischen je 20 Saiblinge in kubischen Behältern aus verzinktem Drahtnetz von $1 / 4 \mathrm{cbm}$ Fassungsraum in 5,10 und $17 \mathrm{~m}$ von demselben Floß aus versenkt. Der tiefste Kasten lag auf Grund auf. Außerdem wurden noch ein Behälter mit 20 Forellen in gleicher Weise in $2 \mathrm{~m}$ Tiefe befestigt. Diese letzteren wurden wohl auch von Ichthyophthirius ergriffen, und arm 28. August waren nur mehr fünf davon vorhanden. Die Saiblinge in größeren Tiefen dagegen hielten sehr gut aus, obwohl, 'wie früher erwähnt, ihre auf der Oberfläche schwimmenden Brüder der Seuche vollkommen zum Opfer gefallen waren. 
In den einzelnen Tiefen fanden sich am 28. August folgende Individuenzahlien und Gewichte iff. je 10 Stück) vor:

$\begin{array}{lccc}\text { Tiefe } & 5 \mathrm{~m} & 10 \mathrm{~m} & 17 \mathrm{~m} \\ \text { Anzahl } & 18 & 17 & 16 \\ \text { Gewicht für 10 Stück } & 6,8 \mathrm{~g} & 6,1 \mathrm{~g} & 4,1 \mathrm{~g}\end{array}$

Der Prozentsatz der eingegangenen Tiere ist also ein relativ geringer. Die Unterschiede im Gewicht sind natürlich nicht allein auf die verschiedene Nahrungsmenge, sondern auch auf die Temperaturdifferenzen zurückzuführen. Immerhin ist es interessant, daß die Fische in dem auf Grund aufliegenden Kasten in $17 \mathrm{~m}$ Tiefe sich gehalten haben und sogar eine nicht unbeträchtliche Gewichtszunahme aufwiesen, während in flachen Behältern, die gleichzeitig mit ebensoviel Forellen beschickt, auf Chara und Eloden Grund der Uferregion ausgelegt wurden, ein rasches Absterben und eine nur geringe Gewichtszunahme erfolgte. Und doch sollte man meinen, daß diese Fische die Möglichkeit hatten, sich von der grundbewonnenden Fauna zu ernähren.

Doch sind diese Vorversuche über die Ernährungsmöglichkeit in verschiedenen Tiefen und auf verschiedenen Boden- und Vegetationsfazies der Uferregion noch lange nicht spruchreif und müssen noch eine Wiederholung nach verbesserten Methoden erfahren. Die auf Bodengrund und im freien Wasser erhaltenen Resultate sind auch nicht direkt vergleichbar, da ja natürlich die ersteren, insbesondere wenn sie sich oberhalb der Sprungschicht befinden, von einer viel größeren Wassermenge passiert werden, als ihrem Rauminhalt entspricht, während die Fische in den letzteren an das Stück Bodengrund, das der Kasten bedeckt, gebunden sind.

Es war daher nur meine Absicht, in dieser Mitteilung auf die Tatsache hinzuweisen, daß in die limnetische Region eines Sees ausgesetzte Salmonidenbrut sich mit dem dort vorhandenen Plankton $z u$ ernähren und $z u$ wachsen vermag, und daß die Uferflucht der planktonischen Crustaceen in der Gewichtszunahme der in verschiedener Ufernähe befindlichen Fische klar zum Ausdruck kommt.

Diese Tatsache scheint mir auch im Hinblick auf die Püttersche Theorie von der Ernährung der Fische durch im Wasser gelöste organische Substanzen einige Beachtung zu verdienen. Nicht, daß ich etwa glaubte, daß Pütters Anschauungen dadurch wider- 
legt werden. Pütter ${ }^{1}$ ) bezieht ja seine Ausführungen nicht auf Salmoniden und führt auch beim Lachs an, daß er während seines Aufenthaltes im Süßwasser, wo er keine Nahrung aufnehme, sein Gewicht verringere, daß also die durch die Kiemen aus dem Wasser aufgenommenen organischen Stoffe nicht ausreichten, seinen Energiebedarf zu decken. Aber vielleicht wären den früher geschilderten ähnliche Versuche mit anderen Fischspezies geeignet, den Wert der Planktonnahrung im Verhältnis zu einem eventuellen Nährwert der gelösten organischen Substanzen annähernd festzustellen. Denn der Gehalt an gelösten Stoffen ist in einem Gewässer in horizontaler Richtung, soweit man bis jetzt weiß, keinen nennenswerten Veränderungen unterworfen, es könnte höchstens unmittelbar am Ufer der Gehalt an organischer Substanz wegen der dort sich abspietenden Zersetzungsprozesse ein größerer sein als draußen im See.

$\mathrm{Ob}$ den hier besprochenen Versuchen in fischereilich-praktischem Sinne irgendein Wert zukommt, das zu beurteilen halte ich mich weder für kompetent noch das vorliegende Beobachtungsmaterial für ausreichend. Ich weiß auch nicht, ob nicht schon anderswo der Versuch gemacht wurde, Forellenbrut ohne Fütterung nur auf Grund des im Wasser eines Sees unter natürlichen Verhältnissen vorhandenen Planktons aufzuziehen, da mir nicht die ganze fischereiliche Literatur zugänglich ist. Sollte dies nicht der Fall sein, so scheinen mir die in Lunz begonnenen Versuche, die in den kommenden Jahren ihre Fortsetzung finden sollen, einer Wiederholung in anderen, planktonreicheren Seen wert zu sein.

Wenn wir in dem an planktonischen Crustaceen verhältnismäßig armen Lunzer See die in der limnetischen Region ausgesetzten Forellen bei relativ sehr dichter Besetzung der Kästen in ungefähr drei Monaten auf das achtfache ihres ursprünglichen Gewichtes. heranwachsen sehen, so wäre zu erwarten, da $\beta$ in nahrungsreicheren Gewässern, wie die Mehrzahl der Seen des Flachlandes es ist, der Zuwachs ein bedeutend größerer sein dürfte. Und es wäre vielleicht auch nicht ganz ausgeschlossen, daß ein Aussetzen von Salmonidenbrut in der vom Plankton bewohnten Region der Seen mittels geeigneter Behälter, womöglich einige Meter unter der Oberfläche, zu einer bequemen Methode der Aufzucht von einsömmrigen Fischen werden könnte.

1) Pūtter, A., Die Ernährung der Wassertiere und der Stoffhaushalt der Gewässer. Jena 1909. 
Das Ergebnis der oben geschilderten Versuche schien mir auch deshalb auffallend, weil man in letzter Zeit geneigt zu sein scheint, weniger dem Plankton im wahren Sinne des Wortes, als vielmehr der Uferfauna die Hauptrolle bei der Ernährung der Fische zuzuschreiben. Diese Anschauung, die z. B. durch genaue Untersuchungen des Darminhaltes gestützt wird, beruht, soweit ich weiß, auf Beobachtungen an älteren Fischen oder an Jungfischen, die in flachen Aufzuchtteichen ohne typisches Plankton ausgesetzt waren, nicht aber auf Untersuchungen des Darminhaltes nur wenige Monate alter Wildfische der Seen. Durch solche müBte festgestellt werden, ob die Salmonidenbrut im Freien den gewaltigen, im Plankton aufgespeicherten Nahrungsvorrat ausnützt oder verschmäht.

$\mathrm{Da}$ sie, darauf angewiesen, sehr wohl gedeihen kann, beweisen die mitgeteilten Versuche. 